\title{
EchoGéo
}

$47 \mid 2019$

Nouvelles géographies de la collecte

\section{À propos de collectes et de collecteurs : un nouveau regard sur le monde}

Jean-Louis Chaléard

\section{Q OpenEdition}

Journals

Édition électronique

URL : https://journals.openedition.org/echogeo/17274

DOI : $10.4000 /$ echogeo. 17274

ISSN : 1963-1197

Éditeur

Pôle de recherche pour l'organisation et la diffusion de l'information géographique (CNRS UMR 8586)

Référence électronique

Jean-Louis Chaléard, «À propos de collectes et de collecteurs : un nouveau regard sur le monde », EchoGéo [En ligne], 47 | 2019, mis en ligne le 21 avril 2019, consulté le 10 août 2021. URL : http:// journals.openedition.org/echogeo/17274; DOI : https://doi.org/10.4000/echogeo.17274

Ce document a été généré automatiquement le 10 août 2021.

EchoGéo est mis à disposition selon les termes de la licence Creative Commons Attribution - Pas d'Utilisation Commerciale - Pas de Modification 4.0 International (CC BY-NC-ND) 


\title{
À propos de collectes et de collecteurs : un nouveau regard sur le monde
}

\author{
Jean-Louis Chaléard
}

1 Échogéo alterne régulièrement des questions générales qui se posent à une échelle mondialisée et des problématiques portant sur des espaces circonscrits, États régions, continents ou sous-continents. Le présent numéro est largement consacré à un sujet qui relève du premier genre: l'activité de "collecte", dans ses formes renouvelées. Outre le dossier sur les « Nouvelles géographies de la collecte», les autres rubriques trimestrielles abordent le sujet. Il nous a semblé important que la revue s'intéresse à une question qui touche à la fois à une analyse des maux de la société de consommation (la production massive de "déchets »), à des formes d'activité en apparence en marge du système capitaliste dominant (telle la cueillette des plantes ou des fruits sauvages), qui peuvent renvoyer à des débats sur la décroissance, sur la marginalité géographique ou sociale et dont l'étude fait l'objet de travaux nombreux actuellement, conduisant à changer notre regard sur une activité longtemps dépréciée ou méprisée.

Dans leur introduction au dossier Sur le champ qu'ils ont dirigé, Lucie Dejouhanet et Rémi de Bercegol justifient le rapprochement de deux activités en apparence dissemblables : le glanage des restes agricoles et la récupération des déchets, utilisant le terme de «collecte", qui qualifie "des processus de ramassage de matières valorisables, disponibles dans des environnements situés ». Le dossier, fort de neuf articles, aborde ainsi des aspects variés, de la collecte de déchets en milieu urbain à la cueillette de plantes dans les forêts ou les friches rurales. Au-delà de leur diversité thématique et géographique (les cas étudiés sont pris en Europe, en Amérique du Sud et du Nord, en Chine), les textes font ressortir quelques questions fortes : sur l'importance de la valorisation de produits délaissés; sur la place de la collecte dans le système capitaliste, alors que l'activité et ses acteurs sont souvent critiqués et dévalorisés ; sur son ambivalence, fait de collecteurs inégalement précaires et aux objectifs différents ; 
sur la façon dont l'étude de cette activité débouche sur une analyse de la société actuelle.

3 Au-delà des différences entre glanage et collecte en milieu urbain, la plupart des articles insistent sur la complexité et la diversité des situations. S. Doyon relève ainsi que la cueillette commerciale d'espèces sauvages au Québec est le fait de deux catégories d'acteurs professionnels et non professionnels, aux pratiques différentes, avec pour enjeu la préservation du milieu et de la durabilité de l'activité. B. Florin et P. Garret étudient les «ferraillous » de la banlieue nord de Paris, personnes en situation de précarité sociale et économique, et qui exercent dans les marges urbaines. Ils montrent comment la collecte des ferrailles s'inscrit dans une économie de la pauvreté, mais n'en alimente pas moins le marché mondial des métaux. L. Springer aborde, dans le cas du nord-ouest de la Chine, autre marge, la cueillette par les populations de montagne, dans leur lutte pour une reconnaissance de leur activité par les autorités.

4 C. Julliand, F. Pinton, R. Garreta et J.-P. Lescure, à partir de l'exemple du projet porté par l'Association française des professionnels de la cueillette, se penchent sur l'organisation professionnelle du secteur dans un but de pérennisation de l'activité et de préservation de l'environnement, face à des collecteurs attirés par les revenus de l'activité et qui ne se soucient pas de sa pérennisation sur le long terme. Dans les cas latino-américains étudiés, M. Durand et F. De Oliveira Neves insistent davantage sur le volet social de la question et montrent l'évolution récente des politiques urbaines: pendant de nombreuses années les villes ont lutté contre les récupérateurs informels de déchets mais cherchent aujourd'hui à reconnaître leur activité dans un but de lutte contre les gaspillages et la pauvreté. Poursuivant l'analyse, M. Rateau et L. Tovar, à partir des exemples de Bogota et Lima, mettent en évidence l'existence de politiques différentes, plus ou moins intégratrices et plus avancées à Bogota. Les deux textes soulignent toutefois les difficultés et les limites actuelles de ces politiques, notamment pour sortir les populations de la pauvreté et les reléguant parfois dans une nouvelle marge.

Les trois derniers articles mettent en évidence l'essor de la cueillette dans les villes européennes et américaines. M.-A. Tarreau, L. Dejouhanet, G. Odonne et M. Palisse, à partir de l'étude de la cueillette des plantes médicinales sauvages en Guyane, montrent que les pratiques utilisées interrogent sur le fonctionnement de sociétés en transition. Ils examinent comment des populations rurales, nouvellement arrivées à Cayenne, adaptent leur pratique à l'environnement urbain. Dans les deux derniers articles, la cueillette est associée à un véritable engagement à la fois social, environnemental et politique. F. Paddeu évoque des cueilleurs new-yorkais engagés, chez lesquels s'affirme une nouvelle manière de voir le monde. L'étude du glanage urbain l'amène à analyser des formes structurelles de pouvoir, d'exclusion, d'inégalités ainsi que des pratiques alternatives au modèle capitaliste dominant. J.Guien parle de son engagement "freegan", de lutte contre le gaspillage alimentaire dans le Nord-Est parisien. Ses pratiques comme son texte s'inscrivent dans une critique de l'économie du gaspillage.

6 Trois textes, dans les autres rubriques trimestrielles, prolongent ou précisent le propos du dossier. Dans la rubrique Sur le métier, M. Faurie, géographe de formation, rend compte de ses pratiques actuelles de glaneur et de cidriculteur en Dordogne et décrit les raisons qui l'ont conduit à cette évolution. Il montre l'exemple d'un genre de vie original, à la campagne, fondé sur la fabrication de produit de qualité à partir d'une activité de cueillette. Ancré dans sa terre, conduisant sa ferme dans un esprit 
scientifique, il n'a pas laissé de côté son expérience de géographe, pratiquant dans une certaine mesure, une géographie active. Le texte de C. Cirelli, R. de Bercegol et B. Florin dans la rubrique Sur l'image donne à voir les « rebuts » dans leur diversité, de la collecte à leur transformation. On y découvre l'inventivité des acteurs, les savoir-faire dont ils font preuve, occupant souvent des lieux délaissés, et l'articulation des acteurs formels et informels. Le Portefolio est l'occasion de diversifier la palette des exemples analysés dans le dossier, avec des images de Turquie, d'Éthiopie, du Kenya... La rubrique Sur l'écrit permet d'élargir la réflexion: d'abord avec l'interview de M. Quet, auteur d'un ouvrage sur les Impostures pharmaceutiques. Médicaments illicites et luttes pour l'accès à la santé dans lequel il se penche sur la controverse liée à la circulation de produits pharmaceutiques illicites; ensuite avec une mise en regard de son livre avec celui de A.-L. Tsing sur Le champignon de la fin du monde. Sur la possibilité de survivre dans les ruines $d u$ capitalisme, récemment traduit en français. Les deux textes s'intéressent à la circulation des produits, aux jeux sur les échelles et la place de leur objet d'étude par rapport au système capitaliste dominant. On retrouve les questions qui courent tout au long du dossier sur la précarité, le rapport entre le légal et l'illégal, le licite et l'illicite...

Deux autres contributions, sur des sujets différents, complètent ce numéro d'Échogéo. Dans la rubrique Sur le métier, F. Di Tursi approfondit l'usage des outils géographiques, déjà abordé dans des numéros antérieurs, en présentant différents emplois de cartographie et des outils géomatiques dans le monde des policiers, des pompiers, des gestionnaires de crise. Au-delà des limites techniques ou liées à des tensions entre différents services, les outils SIG semblent s'insérer totalement dans les missions des différents gestionnaires de crise. Dans la rubrique Sur l'image F. Roussel s'intéresse aux pourtours végétalisés de l'agglomération parisienne qui font l'objet de nombreux aménagements « verts ». L'approche par les photographies met en évidence comment la valorisation écologique sert des usages et des pratiques. La réalisation de croquis, complémentaire, révèle la dimension paysagère où se mêlent des aspects esthétiques et fonctionnels. Le texte permet une réflexion sur le rôle des images dans l'approche des questions sociales ou environnementales. Il renvoie aussi à des débats sur l'écologie, la préservation du milieu, une autre façon de voir et d'appréhender le monde que l'on trouve au cœur des interrogations des articles sur la cueillette et les cueilleurs. 